\title{
Recent Development: Enantio Selective Eextraction in Chiral Separation
}

\author{
Majidhashemi and Mohammad Reza \\ Hadjmohammadi* \\ Department of Chemistry, University of Mazandaran, \\ Babolsar, Iran \\ *Corresponding author: Mohammad Reza \\ Hadjmohammadi, Department of Chemistry, University \\ of Mazandaran, Babolsar, Iran
}

Received: December 07, 2020; Accepted: January 05, 2021; Published: J anuary 12, 2021

\begin{abstract}
This paper discusses the recent development of enantioselective extraction methods. There are several methods in this field. The principles of all of enantioselective extraction methods are presented a focus on twophase extractions, in these methods used a chiral compound that named chiral selector, this compound distinguishes between two chiral isomers in complexation process, then one isomer more captured by chiral selector, and separation processes happen. The chiral selector should dissolve in on phase. In these methods, typical performance data are reported major emphasis on distribution ratio and enantioselectivity value or selectivity factor. In this paper, we introduce types of enantioselective extraction method, and discuss about them with several examples.
\end{abstract}

Keywords: Enantioselective Extraction; Chiral Separation; Liquid-Liquid Extraction; Solid Phase Extraction

\section{Introduction}

Chiral compounds exhibit different properties in biochemical systems, even though they are indistinguishable in most inanimate environments [1]. Two enantiomers of a charily active drug may have dramatically different pharmacological effects, and one of two enantiomers of a chiral pollutant/xenobiotic may be more toxic [2]. In the modern pharmaceutical especially in anti-cancer drugs only one enantiomer has medicinal properties and other enantiomer doesn't have this properties, in other case maybe one enantiomer is useful for body and other one is harmful or it has any effect on body [3]. Noted to this fact that these drugs have harmful effects for body then using racemic mixture for remedy is not effective. Therefore, separation of racemic mixture is necessary for two reasons, one is that harmful effects reduced and other target is economical target, because anticancer drugs are very expensive and using one enantiomer for remedy decreases costs. Hence, there is a great need to develop the technology for analysis and separation of racemic drugs. Current methods of enantiomeric analysis include such non-chromatographic techniques as polarimetry [4], nuclear magnetic resonance [5], isotopic dilution [6], calorimetry [7], and enzyme [8] techniques. The disadvantages of these techniques are the need for pure samples, and no separation of enantiomers is involved. Quantitation, which does not require pure samples, and separation of enantiomers can be done simultaneously by either Gas Chromatography (GC) [9] or High Performance Liquid Chromatography (HPLC) [10]. Chiral HPLC has proven to be one of the best methods for the direct separation and analysis of enantiomers. Chiral separation is often required as the final product purification step after asymmetric synthesis. The two main industrialscale separation methods each have serious drawbacks. Diastereomer crystallization is inflexible and involves solid phase handling [11], and Simulated Moving Bed Chromatography (SMB) is very expensive and produces rather diluted product streams [12]. Therefore, there is a need for a flexible cost-effective separation method suitable for the commercial production scale. Extraction methods are effective, cheep, and flexible method in small and big scale for chiral separation. Chiral selectors play the most important role in the separation efficiency of all enantiomer separation Techniques [13]. In enantioselective extraction, an enantio pure host is used as a chiral selector to bind enantio specifically and reversibly with a racemic substrate. The enantiomer that a complex most strongly with the chiral selector is recovered from the extract and the other enantiomer remains in the initial balk. If the host is confined to one phase in a biphasic system, an enantiomeric separation of the substrate can take place between the two phases in a single step. If the separation is imperfect, a fractional extraction series is required. In this, review we intend to introduce extraction methods that used in chiral separation in the past, and in the end of we will compare these methods together in discussion part.

\section{Liquid-Liquid Extraction}

Liquid-liquid extraction is a commonly used method to extract and separate compounds based on their relative solubilities in two immiscible phases, usually an aqueous phase and an organic solvent phase. It is an extraction of a substance from one liquid into another liquid phase.

\section{Enantioselective Liquid-Liquid Extraction}

In the enantioselective liquid-liquid extraction, usually chiral selectors were dissolved in the organic solvent, and racemic mixture was dissolved in the aqueous phase. Two phases were mixed sufficiently, and (S)-chiral selector and (R)-chiral selector complexes distributed differently in the water phase and the organic phase, respectively. chiral selectors have different chiral recognition abilities of the (R)-form and (S)-form of analyt. As a result, the desired one enantiomer was rich in the organic phase and other enantiomer was rich in the water phase, which allowed a separation of (R) and (S) to some extent.

Calculation of distribution ratio and selectivity: The extent of extraction is characterized by the distribution ratios DR and DS for 
each enantiomer:

$$
\begin{aligned}
& \mathrm{D}_{\mathrm{R}}=([\mathrm{R}] \text { org allforms }) /([\mathrm{R}] \mathrm{aq} \text { allforms }) \\
& \mathrm{D}_{\mathrm{s}}=([\mathrm{s}] \text { org allforms }) /([\mathrm{s}] \mathrm{aq} \text { allforms })
\end{aligned}
$$

The operational selectivity $\alpha_{\text {op }}$ is defined by the ratio of these distribution ratios.

$$
\alpha_{\mathrm{op}}=\mathrm{D}_{\mathrm{R}} / \mathrm{D}_{\mathrm{S}} \quad \text { Assuming } \mathrm{D}_{\mathrm{R}}>\mathrm{D}_{\mathrm{S}}
$$

The enantioselective extraction of 3,5-dinitrobenzoyl-R,S-leucine by a cinchona alkaloid extractant was studied by heeres et al.at 2008 [14]. Tang et al. at 2013 studied separation of $\alpha$-cyclohexyl-mandelic acid enantiomers by enantioselective liquid-liquid extraction in centrifugal contactor separators [15]. Kim et al. at 2013 studied enantioselective liquid-liquid extractions of underivatized general amino acids with a chiral ketone as chiral selector [16]. He et al. did chiral separation of zopiclone using enantioselective liquid-liquid extraction with mandelicacid ester derivatives as chiral selector [17]. Enantio selective liquid-liquid extraction of racemic ibuprofen by l-tartaric acid derivatives as chiral selector was studied by Ren et al. [18]. Enantioselective liquid-liquid extraction of (D, L)-valine using metal-BINAP complex as chiral selector was done by Tang et al. [19].

\section{Biphasic Recognition Chiral Extraction}

In this method used two chiral selectors, one chiral selector uses in aqueous phase and other one solves in organic phase. Chiral selectors have to distinguish between two isomers as on chiral selector captures one isomer specially and other chiral selector is specially host for other isomer. One chiral selector should dissolve in organic phase and other in aqueous phase. The extraction experiments perform in glass tube. Equal volumes of the aqueous and the organic phase were placed together, and shaken sufficiently before being kept in a water bath at a fixed temperature or other conditions to reach equilibrium. After phase separation, the concentrations of enantiomers in each phase were analyzed by HPLC or other techniques. This method is named Biphasic Recognition Chiral Extraction (BRCE).

Efficient enantioseparation of phenyl succinic acid enantiomers by aqueous two-phase system-based biphasic recognition chiral extraction was studied by Jiao et al. [20]. Chiral separation of 2-phenylpropionic acid enantiomers in a biphasic recognition chiral extraction system and continuous separation of pantoprazole enantiomers by biphasic recognition chiral Extraction in centrifugal contactor separators was studied by Tang et al. [21,22]. Chiral separation of amlodipine besilate enantiomers in a biphasic recognition chiral extraction system was studied by Sun et al. [23]. Liu et al. did biphasic recognition chiral extraction of pantoprazole enantiomers; they used $\beta$-cyclodextrin and tartaric acid as chiral selectors [24]. Sunsandee et al experiment enantioseparation of racemic amlodipine by biphasic recognition chiral separation system [25].

\section{Fractional Reactive Extraction}

Fractional reactive extraction is proposed as alternative for diastereomer crystallisation and SMB chromatography. In this method, the formal liquid-liquid extraction process is combined with a reversible enantioselective complexation reaction. To obtain both enantiomers in the desired purity, the fractional extraction scheme is proposed: an additional wash stream will remove the co- extracted undesired enantiomer from the extract. The process has been demonstrated on lab-scale for various enantiomer separations. Furthermore, processes are reported in literature that are essentially fractional liquid-liquid extraction processes, but that appear under other nomenclature. Examples of such related processes are countercurrent distribution or countercurrent chromatography, centrifugal partition chromatography, Craig extraction and counter-current droplet chromatography. A rather large amount of literature is available on single-stage liquid extraction (named 'batch-wise equilibration', 'partitioning experiments' 'distribution', etc) for partial chiral separation. Extraction is often used to evaluate new chiral selectors, but most of these references do not consider reactive extraction as preparative separation technique, nor contain information that can be used directly for an extraction process design.

In this method the wash feed was prepared by dissolving additive materials and racemic mixture was dissolved inappropriate solution to prepare the feeding phase. The organic feed was prepared by dissolving chiral selector in organic solvent. The extraction experiment was performed by starting the engines of all CCSs and starting the extract (organic phase) pump. After starting the extract pump, the CCSs were filled up in order from final Stage to first Stage. After the organic phase outflow from Stage 1, the wash streams (aqueous phase) were started. When the aqueous phase ran from final Stage, the feed pump was started. The concentrations of complex (both isomers with chiral selector) in the raffinate outlet were analyzed using HPLC or other techniques.

Fractional reactive extraction for separation of 4-Nitro-D,Lphenylalanine in centrifugal contactor separators was studied by Tang et al. [26] study on enantioselective reactive extraction of p-hydroxyphenylglycine enantiomers with metal phosphine complexes was done by Tang et al. [27]. Reactive extraction of L-valine using di(2-ethylhexyl) phosphate acid as chiral selector was done by Li et al. [28].

\section{Hallow Fiber Supported Liquid Membrane}

Hollow fibers are an increasingly attractive alternative to conventional packed towers for gas adsorption and for liquid-liquid extractions. The attraction of hollow fibers does not result from large mass-transfer coefficients; indeed, their mass-transfer coefficients are similar to those in conventional equipment. Instead, the attraction of hollow fibers results from their unusually high surface area per volume. This surface area per volume is typically 30 times larger for fibers than for packed towers, and 100 times larger for fibers than for conventional extractors. A Liquid Membrane (LM) is literally a membrane made of liquid. It consists of a liquid phase (e.g. a thin oil film) existing either in supported or unsupported form that serves as a membrane barrier between two phases of aqueous solutions or gas mixtures.

Two homogeneous, totally mixable liquids, namely a donor solution (feed) and a permeate solution (stripping solution) are divided by a third liquid (membrane phase), which is practically insoluble in the donor and permeate solutions. Although there are a few exceptions, the feed and permeate are mostly aqueous phases. The membrane contains a chiral carrier, which selects and chemically binds one select and, increasing its solubility in the membrane phase. The organic membrane phase wets the membrane 
pores of a microporous-hydrophobic membrane, which in contrast to other membrane separation processes, only serves as a septum in this case. Due to their different chemical potentials, the R-select and Sat the pore-opening interface between the donor solution and the organic membrane are extracted from the donor solution into the membrane phase where they undergo chemical bonding and then diffuse to the permeate side. Conditions are also created on the second interface, which favor a transfer process allowing the stripping of the R-substances out of the membrane liquid and their subsequent concentration in the permeate solution. A different $\mathrm{pH}$ in the aqueous feed-phase and the aqueous permeate phase were used in these method. A small quantity of R- and S-select and dissolved physically and diffused unselectively through the membrane.

Chiral separation of (S)-amlodipine via a hollow fiber supported liquid membrane was experimented by Pancharoen et al. they used $\mathrm{O}, \mathrm{O}^{\prime}$-dibenzoyl-(2S,3S)-tartaric acid as chiral selector in liquid membrane [29]. Study of enantioseparation of (R,S)-amlodipine in liquid membrane using $\mathrm{O}, \mathrm{O}^{\prime}$-dibenzoyl-(2S,3S)-tartaric acid as chiral selector was done by Leepipatpiboon et al. [30]. Resolution of racemic $\alpha$-cyclohexylmandelic acid using chiral microemulsion liquid membrane was done by Huang et al. [31].

\section{Solid-Phase Extraction}

Solid-Phase Extraction (SPE) is a sample preparation and separation process by which compounds that are dissolved or suspended in a liquid mixture are separated from other compounds in the mixture according to their physical and chemical properties. Analytical laboratories use solid phase extraction to concentrate and purify samples for analysis. Solid phase extraction can be used to isolate analytes of interest from a wide variety of matrices, including urine, blood, water, beverages, soil, and animal tissue. This method used for chiral separation in several types.

\section{Molecularly Imprinted Polymer}

A Molecularly Imprinted Polymer (MIP) is a polymer that has been processed using the molecular imprinting technique which leaves cavities in polymer matrix with affinity to a chosen "template" molecule. The process usually involves initiating the polymerization of monomers in the presence of a template molecule that is extracted afterwards, thus leaving complementary cavities behind. These polymers have affinity for the original molecule and have been used in applications such as chemical separations, catalysis, or molecular sensors. Fast and cost-effective molecularly imprinted polymer technique has applications in many fields of chemistry, biology and engineering, particularly as an affinity material for sensors, detection of chemical, antimicrobial, and dye, residues in food, adsorbents for solid phase extraction, binding assays, artificial antibodies, chromatographic stationary phase, catalysis, drug development and screening, and byproduct removal in chemical reaction. Molecular imprinted polymers pose this wide range of capabilities in extraction through highly specific micro-cavity binding sites. Due to the specific binding site created in a MIP this technique is showing promise in analytical chemistry as a useful method for solid phase extraction. The capability for MIPs to be a cheaper easier production of antibody/ enzyme like binding sites doubles the use of this technique as a valuable breakthrough in medical research and application. Such possible medical applications include "controlled release drugs, drug monitoring devices, and biological receptor mimetic". Beyond this MIPs show a promising future in the developing knowledge and application in food sciences.

In this method one isomer used for template, after synthesis of polymer template are removed from polymer. This polymer is ready to use for especially extraction of template. This polymer can use in column or bed for chiral separation. As soon as the MIPs placed in racemic mixture template isomer will placed in its position and another one remain in initial solution or mobile phase (in column methods) then separation process will complete.

In recent years $\mathrm{N}$ - $\alpha$-benzyloxy carbonyl glutamic acid isomers were separated in MIP method by Yoshikawa et al., they used polysulfone with aldehyde in structure of fiber [32]. Racemic mixture of 1,1'-binaphthalene-2,2'-diamine was separated by MIP methods in HPLC, Dong et al. used MIP as stationary phase in HPLC column [33]. L-tryptophan, L-valine, L-threonine, S-(-)-ofloxacin isomers were separated by this method by Liang et al. [34]. Imprinted polymers for chiral resolution of $( \pm)$-ephedrine was synthesized by Ansell et al. they used synthesized MIP as stationary phase in supercritical fluid chromatography [35]. Chiral Separation of Amlodipine and its enantiomer on a Molecularly Imprinted Polymer were done by Li et al. [36].

\section{Nanoparticles}

Nanoparticles are of great scientific interest as they are, in effect, a bridge between bulk materials and atomic or molecular structures. A bulk material should have constant physical properties regardless of its size, but at the Nano-scale size-dependent properties are often observed. Thus, the properties of materials change as their size approaches the nanoscale and as the percentage of the surface in relation to the percentage of the volume of a material becomes significant. For bulk materials larger than one micrometer (or micron), the percentage of the surface is insignificant in relation to the volume in the bulk of the material. The interesting and sometimes unexpected properties of nanoparticles are therefore largely due to the large surface area of the material, which dominates the contributions made by the small bulk of the material. For biological applications, the surface coating should be polar to give high aqueous solubility and prevent nanoparticle aggregation. In serum or on the cell surface, highly charged coatings promote non-specific binding, whereas polyethylene glycol linked to terminal hydroxyl or methoxy groups repel non-specific interactions. Multivalent nanoparticles, bearing multiple targeting groups, can cluster receptors, which can activate cellular signaling pathways, and give stronger anchoring. Monovalent nanoparticles, bearing a single binding site. Avoid clustering and so are preferable for tracking the behavior of individual proteins.

In this method chiral selectors are coated on nanoparticle surface, and nanoparticles insert into racemic mixture, then chiral selector captures one isomer especially and other one remain in initial balk. Super magnet collects nanoparticles in corner of sample container and separation process will complete.

Chiral separation of tryptophan isomers was done using nanoparticle methods by Yang et al. they used modified mesoporous silica $\mathrm{Fe}_{3} \mathrm{O}_{4}$ magnetic nanoparticles in this research [37]. Enantioselective separation of chiral aromatic amino acids with 
surface functionalized magnetic nanoparticles was done by Uddin et al. they used carboxymethyl- $\beta$-cyclodextrin as chiral selector for coating nanoparticle surface [38]. Racemic ibuprofen and ofloxacin were separated using bovine serum albumin functionalized magnetic nanoparticles by li et al. [39].

\section{Discussion}

Several extraction methods used for chiral separation. Extraction methods are semi industrial method. In these methods we can separate chiral compounds in $\mathrm{kg}$ scale. Advantages of these methods over other methods are: simplicity, inexpensive, application in large scale and effective. Disadvantage of these methods are: Nonautomatic and Inapplicable for many compounds.

Among these methods liquid-liquid extraction is very simple and fast because this method done by simple tool of laboratory but this method isn't effective for chiral separation because in this methods the two solvents are mixed together but mixing time is short and chiral selector don't have time for well complexation. In result selectivity value in liquid-liquid extraction methods is less than other methods.

Solid phase extractions are more effective than liquid extractions and give more selectivity but these methods are inapplicable in large scale and we can use these methods for micro scale.

\section{References}

1. Y Yue, $X-Y$ Jiang, J-G Yu, K-W Tang. Enantio separation of mandelic acid enantiomers in ionic liquid aqueous two-phase extraction systems. Chem. Pap. 2014; 68: 465-471.

2. JJ. Darrow. Patentability of Enantiomers: Implications for the Pharmaceutical Industry, the Stan. Tech. L. Rev. 2007; 23.

3. M Galanski, A Yasemi, S Slaby, MA Jakupec, VB Arion, M Rausch, AA Nazarov. Synthesis, crystal structure and cytotoxicity of new oxaliplatin analogues indicating that improvement of anticancer activity is still possible. Eur. J. Med. Chem. 2004; 39: 707-714.

4. DM Goodall. Chiral analysis based on polarimetric detection. TrAC Trends Anal. Chem. 1993; 12: 177-184.

5. L Ge, G-YA Tan, L Wang, C-L Chen, L Li, SN Tan, et al. Determination of monomeric composition in polyhydroxyalkanoates by liquid chromatography coupled with on-line mass spectrometry and off-line nuclear magnetic resonance. Talanta. 2016; 146: 107-113.

6. B. Jin, M. Rolle. Joint interpretation of enantiomer and stable isotope fractionation for chiral pesticides degradation. Water Res. 2016; 105: 178186.

7. SL Anderson. Probing Bile Salt Micelle Aggregation and Chiral Guest-Host Binding Using Isothermal Titration Calorimetry. 2016.

8. WC DeLoache, ZN Russ, L Narcross, AM Gonzales, VJJ Martin, JE Dueber et al. An enzyme-coupled biosensor enables (S)-reticuline production in yeast from glucose. Nat. Chem. Biol. 2015; 11: 465-471.

9. V Schurig, M Juza. Analytical separation of enantiomers by gas chromatography on chiral stationary phases, Adv Chromatogr. 2014; 52 117-168

10. S Tong, $\mathrm{H}$ Zhang, M Shen, $\mathrm{Y}$ Ito, J Yan. Enantioseparation of mandelic acid derivatives by high performance liquid chromatography with substituted $\beta$-cyclodextrin as chiral mobile phase additive and evaluation of inclusion complex formation, J. Chromatogr. B. 2014; 962: 44-51.

11. L Ge, Q Zhao, K Yang, S Liu, F Xia. Optical resolution and optimization of ( $R$, S)-propranolol using dehydroabietic acid via diastereomeric crystallization. Chirality. 2015; 27: 131-136.

12. BR Caes, TR Van Oosbree, F Lu, J Ralph, CT Maravelias, RT Raines.
Simulated moving bed chromatography: Separation and recovery of sugars and ionic liquid from biomass hydrolysates. ChemSus Chem. 2013; 6: 20832089.

13. B Chen, Y Du, H Wang. Study on enantiomeric separation of basic drugs by NACE in methanol-based medium using erythromycin lactobionate as a chiral selector, Electrophoresis. 2010; 31: 371-377.

14. B Schuur, JGM Winkelman, HJ Heeres. Equilibrium studies on enantioselective liquid-liquid amino acid extraction using a cinchona alkaloid extractant. Ind. Eng. Chem. Res. 2008; 10027-10033.

15. K Tang, $\mathrm{H}$ Zhang, $\mathrm{P}$ Zhang. Continuous separation of $\alpha$-cyclohexyl-mandelic acid enantiomers by enantioselective liquid-liquid extraction in centrifugal contactor separators: experiments and modeling. Ind. Eng. Chem. Res. 2013; 52: 3893-3902.

16. H Huang, R Nandhakumar, M Choi, Z Su, KM Kim. Enantioselective liquidliquid extractions of underivatized general amino acids with a chiral ketone extractant. J Am Chem. Soc. 2013; 2653-2658.

17. YF Peng, Q He, B Zuo, HB. Niu, TZ Tong, HL Zhao. Enantioselective liquidliquid extraction of zopiclone with mandelic acid ester derivatives, Chirality. 2013; 952-956.

18. Z Ren, Y Zeng, Y Hua, Y. Cheng, Z. Guo. Enantioselective liquid-liquid extraction of racemic ibuprofen by L-tartaric acid derivatives. J. Chem. Eng. Data. 2014; 59: 2517-2522.

19. $K$ Tang, $T$ Fu, $P$ Zhang. Enantioselective liquid-liquid extraction of (D, L) valine using metal-BINAP complex as chiral extractant. J. Chem. Technol. Biotechnol. 2013; 88: 1920-1929.

20. X Chen, J Wang, F Jiao. Efficient enantioseparation of phenylsuccinic acid enantiomers by aqueous two-phase system-based biphasic recognition chiral extraction: Phase behaviors and distribution experiments, Process Biochem. 2015; 50: 1468-1478.

21. K Tang, L Song, Y Liu, J Miao. Enantioselective partitioning of 2-phenylpropionic acid enantiomers in a biphasic recognition chiral extraction system. Chem. Eng. J. 2012; 180: 293-298.

22. Y Wang, K Tang, $P$ Zhang, J Zhou, Y Huang, $P$ Wen, et al. Continuous Separation of Pantoprazole Enantiomers by Biphasic Recognition Chiral Extraction in Centrifugal Contactor Separators, Org. Process Res. Dev. 2014; 19: 1082-1087.

23. P Zhang, G Sun, Y Qiu, K Tang, C Zhou, C Yang. Equilibrium study on enantioselective distribution of amlodipine besilate enantiomers in a biphasic recognition chiral extraction system. J. Incl. Phenom. Macrocycl. Chem. 2016; 85: 127-135.

24. JJ Liu, C Liu, KW Tang, PL Zhang. Biphasic recognition chiral extractionnovel way of separating pantoprazole enantiomers. Chem. 2014; 68: 599607.

25. N Sunsandee, P Rashatasakhon, P Ramakul, U Pancharoen, K Nootong, N. Leepipatpiboon. Selective enantioseparation of racemic amlodipine by biphasic recognition chiral separation system. Sep. Sci. Technol. 2014; 49: 1357-1365.

26. $K$ Tang, $P$ Wen, $P$ Zhang, $Y$ Huang. Fractional reactive extraction for symmetrical separation of 4-nitro-D, L-phenylalanine in centrifugal contactor separators: experiments and modeling. Chirality. 2015: 27: 75-81.

27. X. Feng, K. Tang, P. Zhang, S. Yin. Experimental and Model Studies on Continuous Separation of 2-Phenylpropionic Acid Enantiomers by Enantioselective Liquid-Liquid Extraction in Centrifugal Contactor Separators. Chirality. 2016; 28: 235-244

28. H Bai, K Guo, X Chen, Z Chang, D Li. Reactive extraction of L-valine with di (2-ethylhexyl) phosphate acid. J. Chem. Technol. Biotechnol. 2016; 91 3096-3102.

29. N Sunsandee, N Leepipatpiboon, P Ramakul, U Pancharoen. The selective separation of (S)-amlodipine via a hollow fiber supported liquid membrane: Modeling and experimental verification. Chem. Eng. J. 2012; 180: 299-308.

30. N Sunsandee, P Ramakul, N Thamphiphit, U Pancharoen, N Leepipatpiboon. 
The synergistic effect of selective separation of (S)-amlodipine from pharmaceutical wastewaters via hollow fiber supported liquid membrane. Chem. Eng. J. 2012; 209: 201-214.

31. D Huang, Y Long, W Liu, Y Hong, M Zhong, S Xu. Resolution of racemic $\alpha$-cyclohexyl mandelic acid using chiral micromicroemulsion liquid membrane. 2015.

32. Y Sueyoshi, A Utsunomiya, M Yoshikawa, GP Robertson, MD Guiver. Chira separation with molecularly imprinted polysulfone-aldehyde derivatized nanofiber membranes. J. Memb. Sci. 2012; 401: 89-96.

33. H Dong, Y Wang, Y Ou, J She, $X$ Shen, J Li, et al. Preparation of molecularly imprinted polymer for chiral recognition of racemic 1, 1'-binaphthalene-2, 2'-diamine by HPLC. Acta Chromatogr. 2013; 26: 683-693.

34. J Chen, RP Liang, XN Wang, JD Qiu. A norepinephrine coated magnetic molecularly imprinted polymer for simultaneous multiple chiral recognition, J. Chromatogr. A. 2015; 1409: 268-276.

35. RJ Ansell, JKL Kuah, D Wang, CE Jackson, KD Bartle, AA Clifford. Imprinted polymers for chiral resolution of ( \pm )-ephedrine, 4: Packed column supercritical fluid chromatography using molecularly imprinted chiral stationary phases. J. Chromatogr. A. 2012; 1264: 117-123.

36. ZW Li, X Jia, CM Xu, L Liu, DC Fu. Chiral separation of amlodipine and its enantiomer on a molecularly imprinted polymer-based stationary phase. In: Adv. Mater. Res. Trans Tech Publ. 2013: 36-39.

37. J Wu, P Su, J Huang, S Wang, Y Yang. Synthesis of teicoplanin-modified hybrid magnetic mesoporous silica nanoparticles and their application in chiral separation of racemic compounds. J. Colloid Interface Sci. 2013; 399: 107-114.

38. S Ghosh, TH Fang, MS Uddin, K Hidajat. Enantioselective separation of chira aromatic amino acids with surface functionalized magnetic nanoparticles, Colloids Surfaces B Biointerfaces. 2013; 105: 267-277.

39. Y Fu, T Huang, B Chen, J Shen, X Duan, J Zhang, et al. Enantioselective resolution of chiral drugs using BSA functionalized magnetic nanoparticles. Sep. Purif. Technol. 2013; 107: 11-18. 